\section{Modeling of the Radiation Pattern of a Pair of Thin Circular-Arc Dipoles Over an Infinite Ground Plane}

\author{
Dirk I.L. de Villiers, Member, IEEE, \\ Robert Lehmensiek, Senior Member, IEEE
}

\begin{abstract}
This communication presents a model of the radiation pattern of two circular-arc dipoles above an infinite perfectly electrically conducting ground plane. A Fourier expansion is used to describe the currents on the wires, and thin circular loop antenna theory is used to derive the radiation patterns. The results are compared to Method of Moments simulations, and it is shown that higher order cosinusoidal harmonics are required in the current model to achieve good correlation between the radiation pattern models and the full wave simulations.
\end{abstract}

Index Terms-Antenna radiation patterns, Dipole antennas, Reflector antenna feeds.

\section{INTRODUCTION}

Two parallel dipole antennas, fed in phase and arranged horizontally over a ground plane has been popular as a reflector feed since the configuration was described by Kraus in his classical text on antennas in 1950 [1]. Renewed interest in this configuration has been sparked by the development of a wide band extension of the basic idea - the so called Eleven antenna [2]. This antenna employs a log-periodic array of folded dipole pairs to achieve the desired wide band performance. An extension of this work involves the development of an antenna incorporating circular-arc folded dipoles to improve the $\mathrm{BOR}_{1}$ efficiency [3], and some preliminary results are reported in [4]. A recent paper by Karandikar [5] reports on a study of the radiation patterns of two dipoles above a ground plane for both linear and circularly curved elements. Several reflector feed performance parameters are compared for different heights above the ground plane and separations between the half wavelength elements. A simple analytical formula is used for the linear elements, but full wave simulations are required for the circular elements.

This communication presents a two-harmonic formulation suitable for use in future studies of the far field radiation patterns of center-driven circular-arc dipoles. The formulation includes an infinite perfect electric conducting (PEC) ground plane, which may be omitted if desired. Following the exact integration procedure for the vector potentials of thin circular loop antennas described in [6], a Fourier series representation of the current on the antenna elements is used to calculate the radiation pattern. The modeled radiation patterns are compared to full wave Method of Moments (MoM) solutions calculated using the commercial code FEKO [7]. Two current models are considered, and it is shown that the most accurate radiation

This work was financially supported by EMSS Antennas (Pty) Ltd in Stellenbosch, South Africa and by the National Research Foundation through the South African SKA project.

D.I.L. de Villiers is with the Department of Electrical and Electronic Engineering, Stellenbosch University, Stellenbosch, 7600, South Africa (email: ddv@sun.ac.za)

R. Lehmensiek is with EMSS Antennas (Pty) Ltd. in Technopark, Stellenbosch, 7600, South Africa (e-mail: lehmensk@emss.co.za)

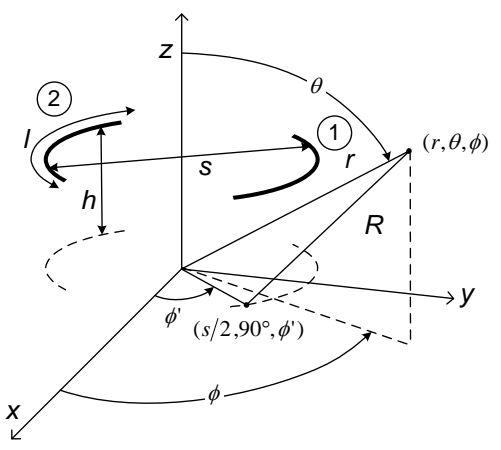

Fig. 1. Geometry of two circular-arc dipole antennas above an infinite perfect electric conducting ground plane at $z=0$. The original antennas are shown as solid lines, and the projection onto the ground plane as dashed lines. The ground plane projection is used to calculate the radiation pattern model, and the original problem is reconstructed using image theory.

patterns are obtained by including higher order cosinusoidal current distributions - especially for longer antennas. A simple model of the required complex higher order modal coefficients is extracted and evaluated, confirming the accuracy of the model.

\section{Theoretical Development}

The basic geometry of the antenna is shown in Fig. 1 along with the standard spherical coordinate system. As usual, source coordinates are primed and field coordinates not. The dipoles, each of length $l$, are circular-arcs with radius $s / 2$ and are arranged symmetrically about the $x=0$ plane at a height $h$ above an infinite conducting ground plane at $z=0$.

Two models for the current on the antennas are considered, where it is assumed that the dipoles are driven in phase at the center of each wire. The first model, termed the classical model, is derived from the well known thin linear dipole current model (given in, for instance, [8]) on the two elements (numbering shown in Fig. 1) as

$$
\begin{aligned}
& i_{s}^{1}\left(\phi^{\prime}\right)= \begin{cases}I_{0} \sin \left[\frac{s \pi}{\lambda}\left(\phi_{0}-\left|\frac{\pi}{2}-\phi^{\prime}\right|\right)\right], & \phi_{p}^{-} \leq \phi^{\prime} \leq \phi_{p}^{+} \\
0, & \text { otherwise, }\end{cases} \\
& i_{s}^{2}\left(\phi^{\prime}\right)= \begin{cases}-I_{0} \sin \left[\frac{s \pi}{\lambda}\left(\phi_{0}-\left|\frac{\pi}{2}+\phi^{\prime}\right|\right)\right], & \phi_{n}^{-} \leq \phi^{\prime} \leq \phi_{n}^{+} \\
0, & \text { otherwise, }\end{cases}
\end{aligned}
$$

with $\phi_{p}^{-}=\frac{\pi}{2}-\phi_{0}, \phi_{p}^{+}=\frac{\pi}{2}+\phi_{0}, \phi_{n}^{-}=-\frac{\pi}{2}-\phi_{0}$ and $\phi_{n}^{+}=$ $-\frac{\pi}{2}+\phi_{0}$. The amplitude of the current is $I_{0}$, the wavelength is denoted by $\lambda$ and $\phi_{0}=l / s$. The second model, termed the harmonic model, consists of any number of harmonics of the fundamental cosinusoidal distribution. The harmonic currents (where the term "harmonic" refers to $\phi$ variations instead of the usual frequency variations) on the respective elements are described by

$$
\begin{aligned}
& i_{m}^{1}\left(\phi^{\prime}\right)= \begin{cases}I_{m} \cos \left[\frac{m \pi}{2 \phi_{0}}\left(\phi^{\prime}-\frac{\pi}{2}\right)\right], & \phi_{p}^{-} \leq \phi^{\prime} \leq \phi_{p}^{+} \\
0, & \text { otherwise, }\end{cases} \\
& i_{m}^{2}\left(\phi^{\prime}\right)= \begin{cases}-I_{m} \cos \left[\frac{m \pi}{2 \phi_{0}}\left(\phi^{\prime}+\frac{\pi}{2}\right)\right], & \phi_{n}^{-} \leq \phi^{\prime} \leq \phi_{n}^{+} \\
0, & \text { otherwise, }\end{cases}
\end{aligned}
$$

where the complex coefficient $I_{m}$ represents the amplitude and phase of the $m$ th harmonic. The total vector currents for the 
respective models are thus

$$
\mathbf{i}_{\mathbf{s}}\left(\phi^{\prime}\right)=\left[i_{s}^{1}\left(\phi^{\prime}\right)+i_{s}^{2}\left(\phi^{\prime}\right)\right] \hat{\mathbf{u}}_{\phi}=i_{s}\left(\phi^{\prime}\right) \hat{\mathbf{u}}_{\phi}
$$

for the classical model, and

$$
\mathbf{i}_{\mathbf{M}}\left(\phi^{\prime}\right)=\sum_{m=1,3, \ldots}^{M}\left[i_{m}^{1}\left(\phi^{\prime}\right)+i_{m}^{2}\left(\phi^{\prime}\right)\right] \hat{\mathbf{u}}_{\phi}=i_{M}\left(\phi^{\prime}\right) \hat{\mathbf{u}}_{\phi}
$$

for the harmonic model, with $M$ the the largest odd harmonic and $\hat{\mathbf{u}}_{\phi}$ the $\phi$-directed unit vector. Even harmonics are not included to ensure that the currents vanish at the endpoints of the wires. For sufficiently large $M$, any current distribution symmetric across the $x=0$ plane may be described by the formulation in (2) and (4), providing for greater freedom and accuracy than the classical model in (1) and (3).

Due to the $2 \pi$ periodicity in $\phi^{\prime}$, and the symmetry of the problem, the current may be expanded in a Fourier sine series as

$$
I\left(\phi^{\prime}\right)=\sum_{n=1}^{\infty} I_{n} \sin \left(n \phi^{\prime}\right) .
$$

It is simple, though tedious, to show that for the classical model

$$
\begin{aligned}
I_{n}^{s} & =\frac{1}{\pi} \int_{-\pi}^{\pi} i_{s}(\phi) \sin (n \phi) d \phi \\
& =\frac{4 I_{0} s \sin \left(\frac{n \pi}{2}\right)}{\lambda\left[(s \pi / \lambda)^{2}-n^{2}\right]}\left[\cos \left(n \phi_{0}\right)-\cos \left(\frac{s \pi \phi_{0}}{\lambda}\right)\right],
\end{aligned}
$$

and, similarly, for the harmonic model

$$
\begin{gathered}
I_{n}^{M}=\sum_{m=1,3, \ldots}^{M} \frac{8 I_{m} \phi_{0} \sin \left(\frac{n \pi}{2}\right)}{\pi\left[(\pi m)^{2}-\left(2 \phi_{0} n\right)^{2}\right]}\left[\pi m \cos \left(n \phi_{0}\right) \sin \left(\frac{m \pi}{2}\right)\right. \\
\left.-2 \phi_{0} n \sin \left(n \phi_{0}\right) \cos \left(\frac{m \pi}{2}\right)\right] .
\end{gathered}
$$

The full radiation patterns for loop currents represented by Fourier cosine series has been developed in [6]. Since only far-field patterns are of interest, the antenna in Fig. 1 may be moved to the $z=0$ plane to present the same problem as the loop current described in [6]. Image theory can be employed to reconstruct the original problem and include the ground plane. The far-field electric field components of (5), when the current is located in the $z=0$ plane, are subsequently given for the sine series used here by

$$
\begin{aligned}
& E_{\theta} \approx \frac{\eta \cot \theta}{2} \frac{e^{-j k_{0} r}}{r} \sum_{n=1}^{\infty} n(j)^{n} I_{n}^{s, M} \cos (n \phi) J_{n}(w),(8) \\
& E_{\phi} \approx-\frac{\eta k_{0} s}{4} \frac{e^{-j k_{0} r}}{r} \sum_{n=1}^{\infty}(j)^{n} I_{n}^{s, M} \sin (n \phi) J_{n}^{\prime}(w),
\end{aligned}
$$

with $k_{0}$ the phase constant, $E_{r} \approx 0$ and $w=\left(s k_{0} / 2\right) \sin \theta$. Superscripts $s$ and $M$ respectively represent the classical and harmonic models in (6) and (7). The argument derivative of the $n$th order Bessel function, $J_{n}(w)$, is represented by the recursive relation

$$
J_{n}^{\prime}(w)=\frac{1}{2}\left[J_{n-1}(w)-J_{n+1}(w)\right] .
$$

The magnetic field components are simply

$$
H_{r} \approx 0, \quad H_{\theta} \approx-E_{\phi} / \eta, \quad H_{\phi} \approx E_{\theta} / \eta
$$

with $\eta$ the free-space impedance.

Using image theory to reconstruct the original problem of two dipoles above a conducting ground plane, the array factor of the pattern is given by

$$
A=\sin \left[k_{0} h \cos (\theta)\right] .
$$

The final radiation pattern function is thus

$$
\mathbf{G}(\theta, \phi)=A\left(E_{\theta} \hat{\theta}+E_{\phi} \hat{\phi}\right)\left[\frac{e^{-j k_{0} r}}{r}\right]^{-1},
$$

to suppress the standard far-field phase and amplitude factors.

\section{COMPARISON With Full WAVE Simulations}

In order to evaluate the accuracy of the classical model in (1), and to determine the unknown complex harmonic weights $I_{m}$ in (2), the model in (13) is fitted on MoM simulation results of several antennas with wire radius of $l / 1000$ over a range of parameters $l, s$ and $h$. The fit is performed by minimizing the cumulative error function defined between simulated (superscript $\mathrm{sim}$ ) and modeled (superscript $\mathrm{mod}$ ) normalized (indicated by a tilde) electric fields defined as

$$
\begin{array}{r}
e r r=\sum_{t=1}^{N_{\theta}} \sum_{p=1}^{N_{\phi}}\left[\left(\left|\tilde{E}_{C O}^{s i m}\left(\theta_{t}, \phi_{p}\right)-\tilde{E}_{C O}^{\bmod }\left(\theta_{t}, \phi_{p}\right)\right|\right)+\right. \\
\left.\left(\left|\tilde{E}_{X P}^{s i m}\left(\theta_{t}, \phi_{p}\right)-\tilde{E}_{X P}^{\bmod }\left(\theta_{t}, \phi_{p}\right)\right|\right)\right] \frac{1}{N_{\theta} N_{\phi}},
\end{array}
$$

where $t$ and $p$ indicate positions in the $\theta$ and $\phi$ vectors of length $N_{\theta}$ and $N_{\phi}$ respectively. For this study $N_{\theta}=45$ and $N_{\phi}=73$ with the fields sampled uniformly in $\theta$ and $\phi$ over the positive $z$ hemisphere. The co-polar (subscript $C O$ ) and cross-polar (subscript $X P$ ) components are calculated using Ludwig's well known third definition [9] from the radiation pattern in (13). Some field cuts of two typical cases are shown in Fig. 2. The effect of including the third harmonic in the current model, which, when normalized to the fundamental harmonic, has values of $I_{3}=0.0564 \angle 159^{\circ}$ in Fig. 2(a) and $I_{3}=0.2957 \angle 159^{\circ}$ in Fig. 2(b), is noticeable mainly in the cross-polar results. The error using the first and third harmonic is reduced to $-72.0 \mathrm{~dB}$ from the $-39.6 \mathrm{~dB}$ obtained by using only the first harmonic in Fig. 2(a) and from $-23.5 \mathrm{~dB}$ to $-71.7 \mathrm{~dB}$ for the longer antenna in Fig. 2(b). The classical model produces accurate co-polar results, with some crosspolar errors noticeable for the longer antenna in Fig. 2(b) where an error of $-36.7 \mathrm{~dB}$ is obtained. Notice that for $l=s=0.5 \lambda$ the classical and harmonic models in (1) and (2) are exactly the same for the fundamental harmonic. The effect of including the fifth harmonic is shown in Fig. 2(c), where the improvement in the harmonic model is obvious for the longer antennas.

Simulations were performed over a wide range of parameters, and some results are shown in Fig. 3. When only the fundamental harmonic is used in the harmonic model the errors increase dramatically as the length of the antennas are increased - especially as the length approaches and exceeds a half wavelength. As expected, using the classical model produces improved results for longer antennas. Including the 


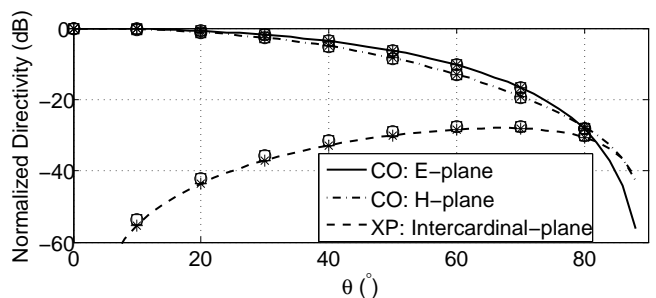

(a)

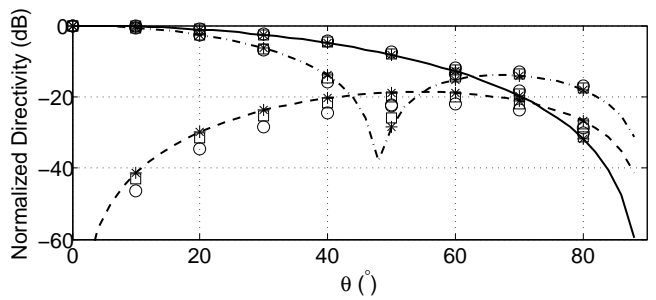

(b)

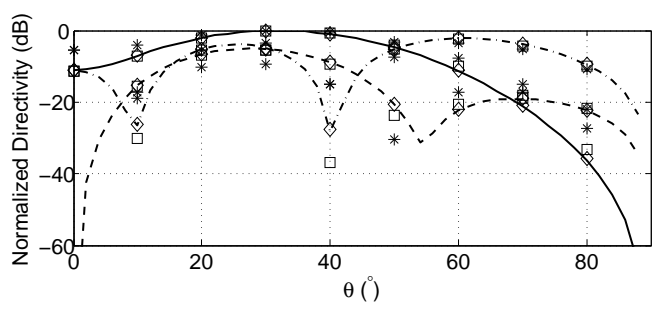

(c)

Fig. 2. Comparison of some field cuts of the simulated (lines) and modeled (markers) field patterns. The circular markers indicate the harmonic model solution with only the first harmonic included in the source current distribution, and the star markers the same model including the first and third harmonics. Square markers indicate the classical model solution. For the plots in (a) $l=s=0.5 \lambda$ and $h=0.25 \lambda$, and for (b) $l=s=0.75 \lambda$ and $h=0.25 \lambda$. In (c) the effect of including the fifth harmonic is shown by diamond markers for long antennas with $l=s=2 \lambda$ and $h=0.25 \lambda$.

third harmonic in the harmonic model improves the error significantly, but an increase in error is still noticed as the length approaches a full wavelength. This may be alleviated by adding further harmonics to the current model. The errors are found to be insensitive for heights between $0.1 \lambda$ and $0.5 \lambda$, and are not shown here. Note also that the very small errors $(-80 \mathrm{~dB}$ and below) are typically machine precision dependant, and not necessarily a function of the model accuracy.

\section{HaRmonic CoefFicient Model}

Section III compares the modeled radiation patterns of two circular-arc dipole current models to simulated results, and it is seen that, for longer antennas, the harmonic model including at least the first and the third harmonics is required for good correlation between results. The amplitude and phase of the required third order harmonic, normalized to the fundamental, required to obtain the results in Fig. 3(c) is shown in Fig. 4. It is clear that the amplitude and phase of the coefficient is largely invariant to changes in the spacing (similar results hold for the height - not shown), and monotonically increase with length. This result is expected, and a simple quantitative model may be easily derived from the data in Fig. 4. Taking the median value over all available spacings, with $h / \lambda=0.25$ taken as a representative case for heights between $0.1 \lambda$ and

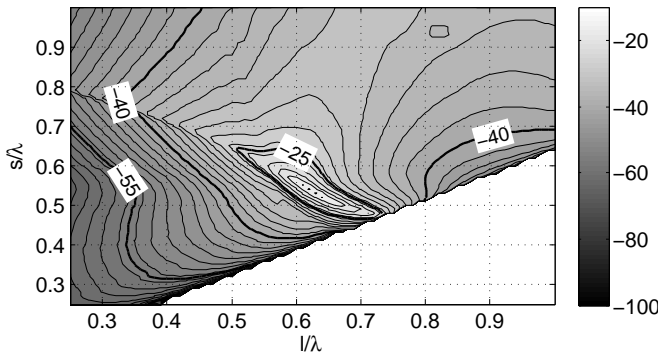

(a)

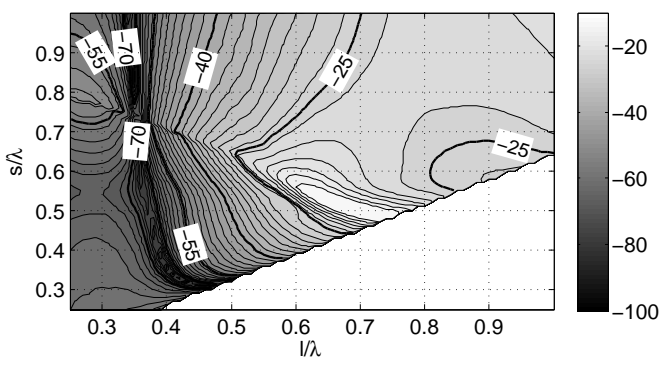

(b)

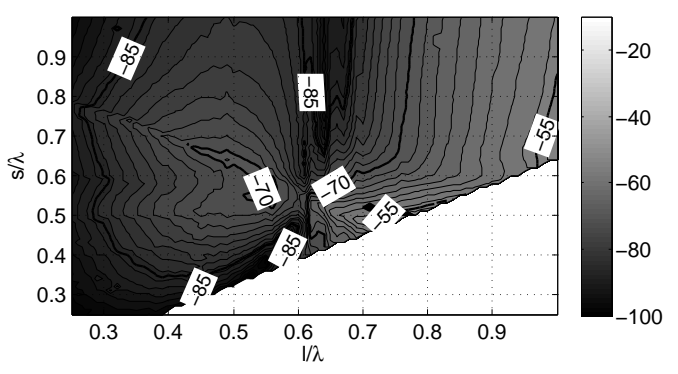

(c)

Fig. 3. Error values (dB) for the classical model in (a), for only the fundamental harmonic in the harmonic model in (b) and for including the third harmonic in the same model in (c). The height is $h=0.25 \lambda$ in all cases.

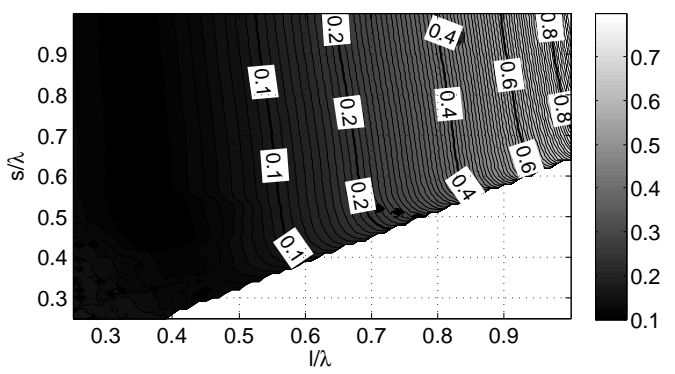

(a)

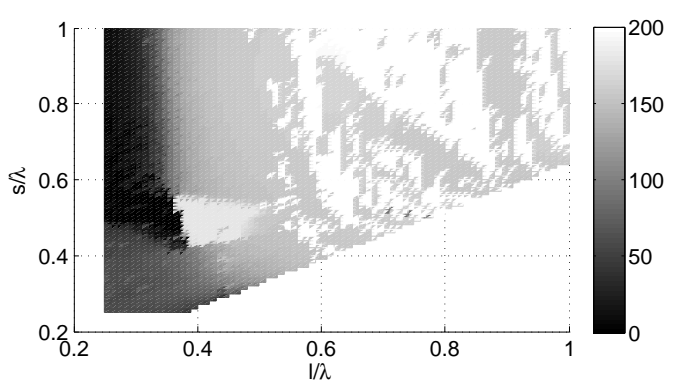

(b)

Fig. 4. Third harmonic amplitude (a) and phase (in degrees) (b) normalized to the fundamental harmonic which minimizes (14). The height is $h=0.25 \lambda$ in all cases. 


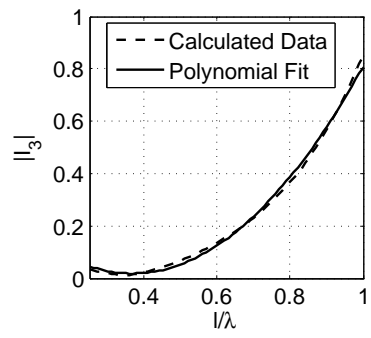

(a)

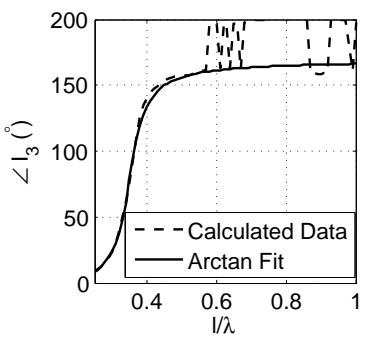

(b)

Fig. 5. Comparison of the simulated results and the empirical model in (15) for the amplitude in (a) and phase in (b), normalized to the fundamental harmonic, which minimizes (14). The height is $h=0.25 \lambda$ in all cases. The calculated data is obtained as the median over spacings in Fig. 4.

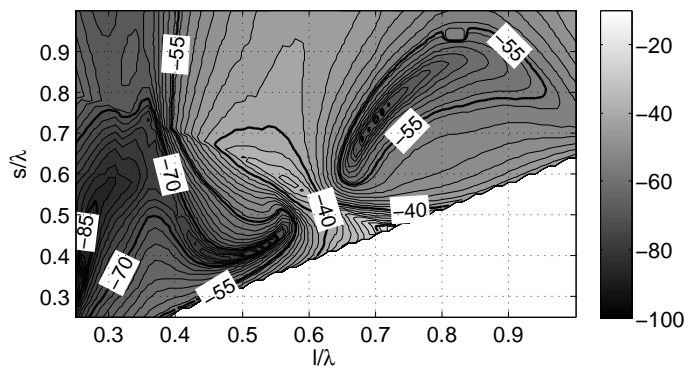

Fig. 6. Error values $(\mathrm{dB})$ when using third harmonic model suggested in (15). The height is $h=0.25 \lambda$ in all cases.

$0.5 \lambda$ (the effect of the height is modeled adequetely by the array factor in (12)), and thus creating a function only in $l / \lambda$, an empirical model of the amplitude and phase of $I_{3}$ is derived as

$$
\begin{aligned}
& \left|I_{3}(l / \lambda)\right|=1.97(l / \lambda)^{2}-1.45(l / \lambda)+0.28, \\
& \angle I_{3}(l / \lambda)=\tan ^{-1} 28.5[(l / \lambda-0.35)]+1.39,
\end{aligned}
$$

with the angle given in radians. All higher harmonics (ie. $\left.I_{m}, m \geq 5\right)$ are zero. The resultant comparison between the model in (15) and the values obtained from the simulations is shown in Fig. 5. The erratic behavior of the phase for longer antennas observed in Figs. 4(b) and 5(b) is due to a flat gradient for the error in (14) with respect to the angle encountered in this region. The error levels achieved using the model in (15) is shown in Fig. 6. Comparison with Fig. 4(a) reveals that the empirical harmonic model produces errors around $15 \mathrm{~dB}$ smaller than those produced by the classical model. For antennas shorter than a wavelength the empirical harmonic model described by (2), (4) and (15) is thus preferred. Longer antennas will require additional harmonics to obtain similar error performance. It is worth noting that the diagonal lines at the edge of the plotted data in Figs. 3, 4, and 6 correspond to configurations with $\phi_{0}=90^{\circ}$ implying closed loop antennas which are not typically used here, and the large errors in that region are therefore of diminished significance.

A wider set of parameters were investigated to find the worst case performance of the suggested model in the region bounded by

$$
h / \lambda \in[0.1,0.5] ; \quad s / \lambda \in[0.25,1.0] ; \quad l / \lambda \in[0.25,1.0] .
$$

At $h / \lambda=0.15, s / \lambda=0.60$, and $l / \lambda=0.55$ the error value using (15) is $-29.3 \mathrm{~dB}$ compared to the $-18.9 \mathrm{~dB}$ obtained

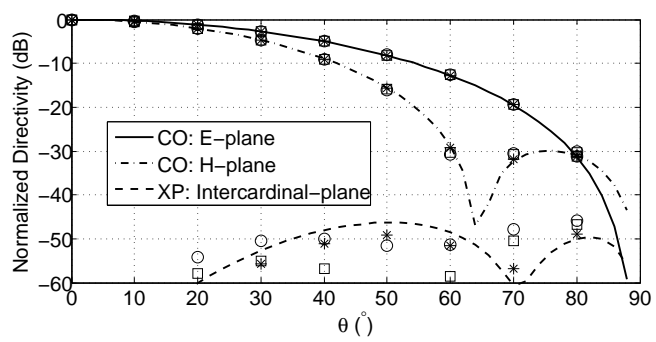

Fig. 7. Comparison of some field cuts of the simulated (lines) and modeled (markers) field patterns. The circular markers indicate the harmonic model solution with only the first harmonic included and the star markers the empirical harmonic model including the first and third harmonics calculated using (15). Square markers indicate the classical model solution. Antenna parameters are $l=0.55 \lambda, h=0.15 \lambda$ and $s=0.60 \lambda$ corresponding to the worst error performance obtained in the region defined by (16).

by using only the fundamental harmonic. The error for the classical model is $-20.6 \mathrm{~dB}$. A plot of the fields for this worst case is given in Fig. 7, where the improvement in the crosspolar field results is obvious.

\section{CONCLUSION}

This note presented the derivation of a model for the radiation pattern of two circular-arc dipoles fed in phase over an infinite PEC ground plane. The current model required for the field calculations allow for higher order harmonics, and the effect of including these is investigated by comparisons with full wave simulations for a wide range of antenna geometry parameters. It was shown that higher order harmonics are required to accurately model the radiation pattern of longer antennas, and that very high levels of correspondence between the modeled and simulated patterns may be achieved. Finally a simple empirical model is derived for the third harmonic coefficient which is valid within a wide region of antenna parameters. It should be stressed here that practical effects such as those caused by a finite size ground plane are not included, and if these are required the model should be augmented by diffraction theory.

\section{REFERENCES}

[1] J. D. Kraus, Antennas. McGraw-Hill, 1950, ch. 11, pp. 309-311.

[2] R. Olsson, P.-S. Kildal, and S. Weinreb, "The eleven antenna: A compact low-profile decade bandwidth dual polarized feed for reflector antennas," IEEE Trans. Antennas Propag., vol. AP-54, no. 2, pp. 368 - 375, Feb. 2006.

[3] P.-S. Kildal, "Factorization of the feed efficiency of paraboloids and cassegrain antennas," IEEE Trans. Antennas and Propag., vol. AP-33, no. 8, pp. 903-908, Aug. 1985.

[4] J. Yin and J. Yang, "A 2-5 GHz circular eleven antenna with improved BOR1 efficiency," in IEEE Int. Symp. Antennas and Propag. (APSURSI), Jul. 2011, pp. $1247-1250$.

[5] Y. Karandikar, "Pattern studies of two parallel dipoles above ground plane in eleven configuration as feed for reflector antenna," IEEE Antennas Wireless Propagat. Lett., vol. 9, pp. 558 -561, 2010.

[6] D. Werner, "An exact integration procedure for vector potentials of thin circular loop antennas," IEEE Trans. Antennas Propag., vol. AP-44, no. 2, pp. $157-165$, Feb. 1996.

[7] EM Software \& Systems - S.A. (Pty) Ltd, Stellenbosch, South Africa. [Online]. Available: http://www.feko.info

[8] C. A. Balanis, Antenna Theory, 3rd ed. Hoboken, NJ, USA: John Wiley \& Sons, 2005, ch. 4, p. 170

[9] A. Ludwig, "The definition of cross polarization," IEEE Trans. Antennas Propag., vol. AP-21, no. 1, pp. 116 - 119, Jan. 1973. 